Moreover, reclassification does not seem to be happening either. If one oesophageal cancer is now being classified as another, the anatomical distribution of all cancers along the oesophagus should remain unchanged. However, there is increased incidence in the lower third of the oesophagus, where adenocarcinomas most commonly occur. The authors also rule out reclassification of gastric adenocarcinoma, as the number of cases of this disease also rose across the period. They conclude that the apparent rise in the incidence of oesophageal adenocarcinoma is real, and that the trend is due to increased prevalence of an as-yetunidentified risk factor.

Helen Dell

(2) References and links ORIGINAL RESEARCH PAPER Pohl, H. \& Welch, H. G. The role of overdiagnosis and reclassification in the marked increase of esophageal adenocarcinoma incidence. J. Natl Cancer Inst. 97, 142-146 (2005)

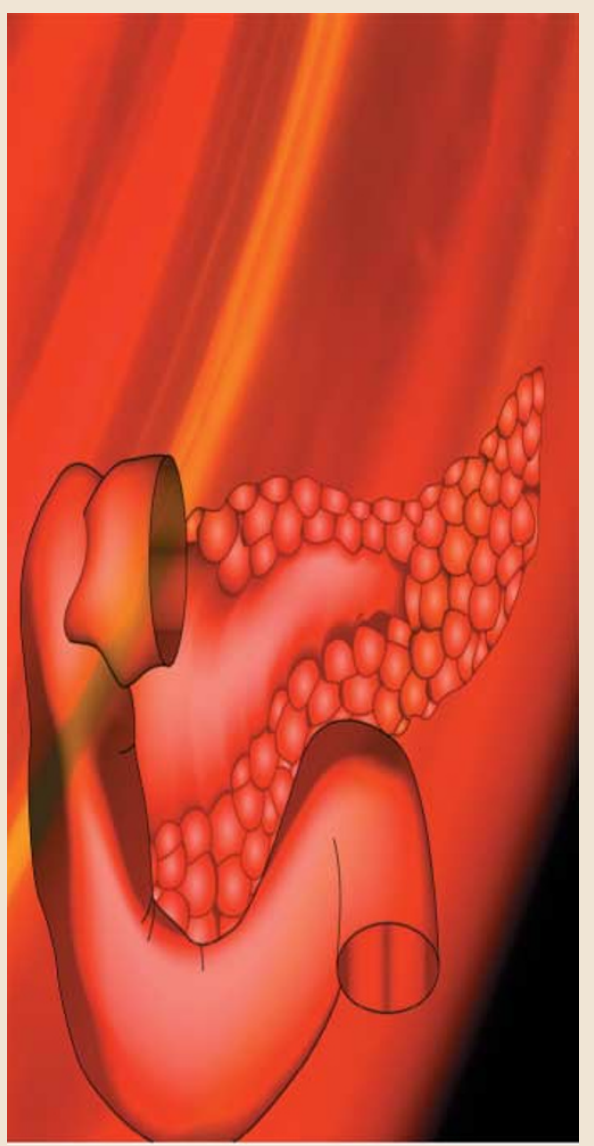

LEUKAEMIA

\section{Space shuttle}

Acute myelogenous leukaemia (AML) is the most common form of leukaemia, but as there are so many subgroups of AML, it is a challenge to select the most effective course of therapy. In the 20 January issue of New England Journal of Medicine, Brunangelo Falini et al. report that the intracellular location of the nucleophosmin (NPM) protein can be used to characterize a large subgroup of patients with AML, and to predict response to therapy.

In normal cells, NPM shuttles between the nucleus and cytoplasm, and is most frequently found in the nucleolus. It is thought to have a tumour-suppressor function, and is a molecular chaperone that might regulate the ARF-p53 pathway. However, it also acts oncogenically in some leukaemias and lymphomas, as the NPM gene is a partner in cancer-associated translocations. In this study, the authors investigated the subcellular location of NPM in 591 specimens from patients with AML, with a view to discovering whether this might affect its tumour-suppressor function.

They found that in 208 of the samples (35\%), NPM was located in the cytoplasm (NPMc+). This pattern was observed across all of the different leukaemia FAB (French-AmericanBritish) subtypes tested, usually associated with CD34 negativity, but was only seen in cells from patients with primary AML. In secondary AML cells, NPM localization was exclusively nuclear. Significantly, $85 \%$ of patients with NPMc+ had normal karyotypes, compared with $27 \%$ of patients with NPMc-. So, cytoplasmic NPM characterizes a distinct subgroup of patients with AML who have a normal karyotype - a group that was previously difficult to stratify.

Does the location of the NPM protein affect the response to therapy? The association was investigated in 126 patients with normal karyotypes for whom NPM immunostaining and clinical information were known. A multivariate logistic-regression model showed that a lower white-cell count and cytoplasmic localization of NPM are independent prognostic factors for a complete remission.

The authors next investigated whether NPM was mutated in the cases in which the protein was cytoplasmic. The characteristic fusion proteins were not found, but sequencing revealed mutations in exon 12 in all but one case of NPMc+ disease. Although these mutations were diverse, they all resulted in frameshifts that altered the carboxy-terminal region of the

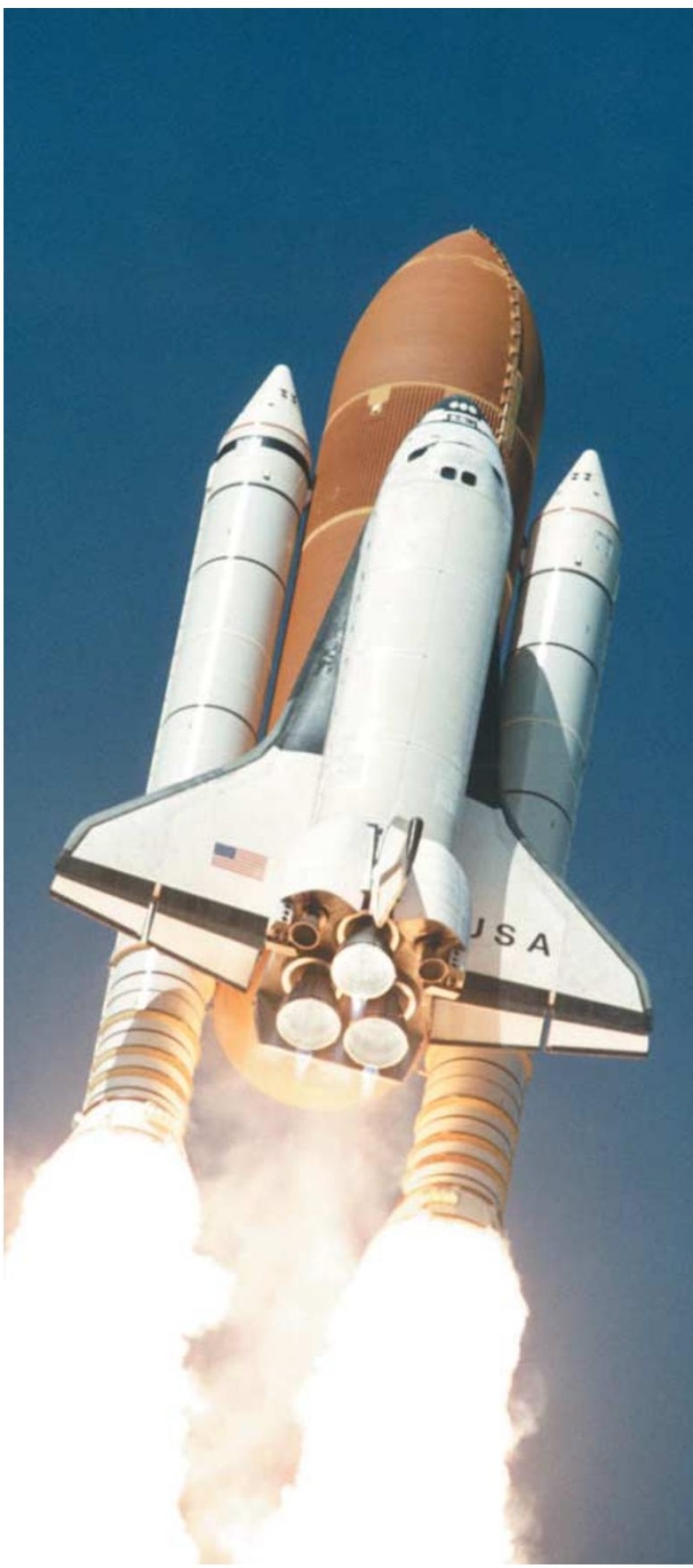

protein. Transient expression of these mutant proteins in NIH-3T3 cells resulted in their cytoplasmic localization, whereas a tagged form of the wild-type protein remained in the nucleolus. These mutations might therefore directly cause the altered distribution.

Emma Greenwood

NPG Executive Editor, Leukemia

(2) References and links

ORIGINAL RESEARCH PAPER Falini, B. et al. Cytosplamic nucleophosmin in acute myelogenous leukemia with a normal karyotype. N. Engl. J. Med. 352, 254-266 (2005) 